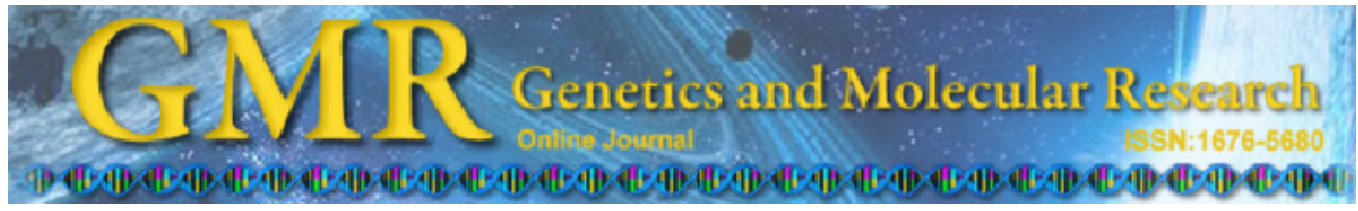

Review

\title{
An overview of odorant-binding protein functions in insect peripheral olfactory reception
}

\author{
J. Fan ${ }^{1,2}$, F. Francis ${ }^{2}$, Y. Liu ${ }^{3}$, J.L. Chen ${ }^{1}$ and D.F. Cheng ${ }^{1}$ \\ ${ }^{1}$ State Key Laboratory for Biology of Plant Diseases and Insect Pests, \\ Institute of Plant Protection, Chinese Academy of Agricultural Sciences, \\ Beijing, China \\ ${ }^{2}$ Functional and Evolutionary Entomology, Gembloux Agro-Bio Tech, \\ University of Liege, Gembloux, Belgium \\ ${ }^{3}$ College of Plant Protection, Shandong Agricultural University, \\ Shandong, China \\ Corresponding authors: J.L. Chen / D.F. Cheng \\ E-mail: jlchen@ippcaas.cn / dfcheng@ippcaas.cn
}

Genet. Mol. Res. 10 (4): 3056-3069 (2011)

Received April 1, 2011

Accepted September 7, 2011

Published December 8, 2011

DOI http://dx.doi.org/10.4238/2011.December.8.2

\begin{abstract}
Insect olfactory perception involves many aspects of insect life, and can directly or indirectly evoke either individual or group behaviors. Insect olfactory receptors and odorant-binding proteins (OBPs) are considered to be crucial to insect-specific and -sensitive olfaction. Although the mechanisms of interaction between OBPs or OBP/ligand complex with olfactory receptors are still not well understood, it has been shown that many OBPs contribute to insect olfactory perception at various levels. Some of these are numerous and divergent members in OBP family; expression in the olfactory organ at high concentration; a variety of combinational patterns between different OBPs and ligands, but exclusive affinity for one OBP to specific binding ligands; complicated interactions between OBP/ligand complex and transmembrane proteins (olfactory receptors or sensory neuron membrane proteins). First, we review OBPs' ligand-binding property based on OBP structural research and ligandbinding test; then, we review current progress around the points cited above
\end{abstract}


to show the role of such proteins in insect olfactory signal transmission; finally, we discuss applications based on insect OBP research.

Key words: Insect olfaction; OBP/ligand complex;

Odorant-binding proteins; Olfactory signal transmission

\section{INTRODUCTION}

Insects are different from higher animals. They depend on olfaction to estimate overall situation of habitats, which is essential for their survival and reproduction, whereas the animals use their highly developed vision to define the outer world. Insect olfactory perception involves individual aspects such as the spotting of food, host, mate or prey, and group communication aspects such as aggregation and avoidance (intraspecific or interspecific). Besides, the recognition of other signals around the habitat environment, which may evoke no behavioral responses, helps insects to distinguish, comprehend and estimate the overall situation. For example, the aphid alarm pheromone (E)- $\beta$-farnesene (EßF) is secreted by at least 16 aphid species (Francis et al., 2005a) but to which the Brevicoryne brassicae does not show their aposematism. Therefore, it has been suggested that $\mathrm{E} \beta \mathrm{F}$ role is to distinguish conspecifics from host plant rather than as an alarm signal by B. brassicae (Francis et al., 2005b). As a huge and diverse group, insects have been estimated to have 4-5 million species (Novotny et al., 2002) and occupy numerous different habitats. Adaptive evolution has resulted not only in their morphological variation but also in their organ functional specialization. For example, insect antennas are grouped in more than 10 basic morphological types but have a common function, olfaction.

Lots of sensilla are located on the surface of the insect antennae with olfactory neurons being protected inside. There are 1-4 dendrites per olfactory sensilla and they are immersed in the hemolymph. Therefore, to trigger the olfactory signal transduction, ligands in the habitat should pass through the hemolymph to stimulate specific dendrites.

Indeed, insects have diverse olfactory organs and a different mechanism of peripheral signal inception and processing, but the resemblances in neuroanatomical logic and physiological coding properties compare with those in mammals, which have been reviewed by Silbering and Benton (2010). Also, there is a comparable function among members in their relative stages of olfactory signal transduction pathways. For example, although odorant-binding proteins (OBPs) in mammals and insects are not homologous, they are both expressed in high concentration around olfactory dendrites, and play a critical role at the first step of olfactory signal transmission. Recently, it has been demonstrated (Buck and Axel, 1991; Benton et al., 2006) that the locating patterns of olfactory receptors (ORs) and the olfactory signal transduction mechanisms between mammals and insects are alien (Neuhaus et al., 2005; Benton et al., 2006; Sato et al., 2008; Wicher et al., 2008). However, only their physiological function is the same, transforming an extracellular chemical signal into an intracellular electronic signal. Then, signals from dispersive olfactory neurons are centralized into a primary processing system, called the olfactory bulb in mice (Friedrich and Korsching, 1997; Mori et al., 1999; Uchida et al., 2000) and antennal lobe, which is analogous to the vertebrate bulb in the fruit fly (Gao et al., 2000; Vosshall et al., 2000). Numbers of glomeruli working in this primary processing system are formed by mitral cells in mice or projection neurons in the fruit fly (Hildebrand and Shepherd, 1997; Strausfeld and Hildebrand, 1999). They concentrate the same olfactory signals from receptors and send them separately to a higher central nervous system. The fruit fly has about 50 (Laissue and Vosshall, 2008) 
glomeruli, which is much less than 1800-2400 glomeruli in mammals depending on the species. The simpler olfactory system of insects is still worth being considered as a model to investigate and compare the regulation of olfactory perception with other organisms.

In insects, some functional components are involved in odor recognition and transduction such as OBPs and ORs. Insect-specific and -sensitive olfactory responses are widely considered to contribute through a combinatorial action of these two protein families as two odorants filters for other flying animals. This paper focuses on the OBP of insect olfactory signal transduction system. We attempt to explain how OBPs work as the first filter for odor recognition and trigger the olfactory perception.

Although OBPs have been identified as the first protein family involved in the olfactory system since 1980s (Vogt and Riddiford, 1981; Pelosi et al., 1981, 1982), scientists could only find few ways to explain the mechanism of olfactory conception during the decade with this isolated protein. This was the situation until the adjacent component, the OR, was identified in mammals (Buck and Axel, 1991). Later, insect ORs were characterized as G protein-coupled receptor (GPCR) according to the mammal receptor (Clyne et al., 1999; Vosshall et al., 1999) in Drosophila, but it was finally revised by Benton et al. (2006) as a different protein family.

To date, more than 300 putative OBP genes have been registered (Zhou et al., 2010a) in GenBank ${ }^{\circledR}$ at the National Center for Biotechnology Information (NCBI) and many OBPs from at least eight orders have been identified (Pelosi et al., 2006) (Table 1).

Table 1. Number of odorant-binding proteins (OBPs) annotated from insect genomes.

\begin{tabular}{llcl}
\hline Species & Common name & OBPs & References \\
\hline Drosophila melanogaster & Fruit fly & 51 & Hekmat-Scafe et al., 2002 \\
Anopheles gambiae & Malaria mosquito & 66 & Xu et al., 2003; Zhou et al., 2008 \\
Aedes aegypti & Yellow fever mosquito & 66 & Zhou et al., 2008 \\
Bombyx mori & Silkworm moth & 45 & Gong et al., 2009 \\
Tribolium castaneum & Red flour beetle & 46 & Foret and Maleszka, 2006 \\
Apis mellifera & Honey bee & 21 & Foret and Maleszka, 2006 \\
Acyrthosiphon pisum & Pea aphid & 15 & Zhou et al., 2010a \\
Culex quinquefasciatus & Southern house mosquito & 53 (classic OBP only) & Pelletier and Leal, 2009 \\
\hline
\end{tabular}

Supplementary base on Zhou et al. (2010a).

OBPs have been divided into four groups according to their primary protein sequences (Hekmat-Scafe et al., 2002; Xu et al., 2003; Pelosi et al., 2006; Zhou et al., 2010a) (Table 2). They are classical OBPs, Plus-C OBPs, which were recently identified in Acyrthosiphon pisum as well as expressed in dipteran insects (Zhou et al., 2010b), Minus-C OBPs and Atypical OBPs, which are only found in blood-sucking insects.

Table 2. Classification of odorant-binding proteins (OBPs) from insects

\begin{tabular}{lcll}
\hline Classification & Cysteine residue & Other description & Example \\
\hline Classical OBPs & 6 & $\begin{array}{l}3 \text { disulfide bonds formed by } \\
6 \text { cysteine residues } \\
\sim 14 \mathrm{kDa}\end{array}$ & $\begin{array}{l}\text { BmorPBP } \\
\text { (Vogt and Riddiford, 1981) }\end{array}$ \\
\hline Plus-C OBPs & $6+2 / 3$ & $\begin{array}{l}1 \text { highly conserved proline residue } \\
\text { at least } 2 \text { conserved cysteines } \\
17-25 \mathrm{kDa}\end{array}$ & $\begin{array}{l}\text { AgamOBP48 Putative Plus-C alignment GenBank } \\
\text { accession No., ALIGN_000581 (Zhou et al., 2004a) }\end{array}$ \\
\hline Minus-C OBPs & $<6$ & - & $\begin{array}{l}\text { Drosophila Obp99a, Obp99b and Obp99d } \\
\text { (Hekmat-Scafe et al., 2002) }\end{array}$ \\
\hline Atypical OBPs & $\geq 6$ & $\begin{array}{l}\text { a long C-terminus } \\
\text { up to } 38 \mathrm{kDa}\end{array}$ & Anopheles OBP35 (Xu et al., 2003) \\
\hline
\end{tabular}


Adaptive evolution in diverse habitats with diverse odor molecules has resulted in a large OBP family with variant proteins (Hekmat-Scafe et al., 2002). Most of insect-OBP sequences are divergent among different orders, even less than $20 \%$ identity from different insect genera (Zhou et al., 2010a). OBP phylogenetic relationship from several model insect species shows a very restricted conservation between different orders (Zhou et al., 2010a). Therefore, it seems that homology cloning is not an effective tool to obtain OBPs among species (Jacobs et al., 2005), which is similar to insect classic olfactory receptor cloning. Using available insect genome information is still the prior option for identifying OBPs.

Diversity of OBPs indicates they distinguish between different ligands or bind to the same ligand in different ways. In contrast, very few highly conserved OBPs indicate common but crucial physiological function in species. LUSH is a pheromone-binding protein (PBP) that prevents sequence conservation from orders, and is required for 11-cis vaccenyl acetate (cVA)-induced behavior and normal cVA sensitivity of the T1 neurons in D. melanogaster (Kim et al., 1998; Xu et al., 2005; Ha and Smith, 2006; Kurtovic et al., 2007; Jin et al., 2008; Laughlin et al., 2008). Further investigation is of interest on this conserved protein in other species. As another example, it has been demonstrated in some OBPs in aphids that the differences of sequences were limited to no more than 2-3 amino acids among species (Qiao et al., 2009). OBP3 is one of these OBPs, which was first identified in A. pisum (Apis OBP3) and has an affinity to E $\beta \mathrm{F}$. This is the first and only identified component involved in insect $\mathrm{E} \beta \mathrm{F}$ perception to date. Either as the alarm pheromone to aphids or as the kairomone to natural enemies, $\mathrm{E} \beta \mathrm{F}$ is one of the shared semiochemicals released by most of aphid species and plays an important role in their chemical communication. This suggests that OBP homology cloning could work on OBPs, especially the common OBPs shared by insects.

\section{THE BINDING PROPERTIES OF OBPS}

As a binding protein expressed by a multigene family, insect OBPs have an overwhelming and common feature, namely their binding activity. Proper designed recombinant insect OBPs expressed in the heterologous system, being identical to natural OBPs in vivo, allow ligand binding assays in vitro. Taking a panoramic view of the OBP-binding test history, three OBP-binding approaches have contributed to the study of OBP-binding affinity with different ligands. However, drawbacks as well as advantages in each of the three methods have been summarized in excellent reviews (Tegoni et al., 2004; Pelosi et al., 2006).

Analysis on the protein caught by tritium-labeled pheromone (E,Z)-6,11-hexadecadienyl incubated with Antheraea polyphemus antennal extract identified the first insect OBP (Vogt and Riddiford, 1981). This method, based on a radioactive-labeled ligand, has been popular for the binding of radioactive pheromone or radioactive photoaffinity. The labeled structural analog shows direct evidence of olfactory specificity in vivo and does not require purified OBPs. On the other hand, radioactivity loss through electrophoresis renders it impracticable to lead to a final conclusion.

Fluorescence-binding tests are currently being chosen for OBPs as a binding measurement, and could be indirectly evaluated by intensity of fluorescence under equilibrium, besides it is simple, safe and fast. Although it requires a fluorescent probe with a binding affinity, tests with OBP could be competed and replaced by ligand. This technique was first performed in insects to demonstrate unspecific binding capacity of PBPs with several ligands 
in A. polyphemus and Mamestra brassicae through a competitive experiment of fluorescent probe, 1-amino-anthracene (AMA), and ligands (Campanacci et al., 2001). Identification of E $\beta$ F-binding protein (Apis OBP3) in A. pisum is another remarkable competitive experiment and example of another widely used probe, N-phenyl-1-naphthylamine (1-NPN), and EßF or its structural analogs to Apis OBP3 (Qiao et al., 2009). Regarding the binding with some ligands, Trp37 has been proposed to be directly involved in change of fluorescence (Campanacci et al., 2001; Bette et al., 2002; Leal et al., 2005), also inducing significant intrinsic fluorescence change, by which one more detail, based on three ApolPBP1-binding ligands, has revealed that there are different interaction modes between ApolPBP1 and the three ligands, (E6,Z11)-hexadecadienal (6E,11Z-16:Al), (E6,Z11)-hexadecadienyl-1-acetate (6E,11Z-16:Ac) and (E4,Z9)-tetradecadienyl-1-acetate (4E,9Z-14:Ac) (Bette et al., 2002).

Another method called cold-binding assay, performed by Leal et al. (2005), shows its unique advantages, which are multiple ligand competitive experiments for best ligand identification and the availability of more accurate determination of constant dissociation based on accurate measurements of the concentrations of free or bound ligand and protein. The binding complex is separated from free ligand filtrate by rapid ultrafiltration. Then, an evaluation of the extraction of bound ligands released from the complex by gas chromatography-mass spectrometry (GC-MS) analysis is performed.

\section{THE THREE-DIMENSIONAL (3-D) STRUCTURE OF OBPs}

Binding tests show unspecific binding properties of OBPs with ligands at times, which can be better understood by further studies at a microscopic level. For example, only 4E,9Z14:Ac of the three ApolPBP1-binding ligands induces a conformational change, which is monitored by circular dichroism measurements on ApolPBP1 secondary structure (Mohl et al., 2002). Microscopic study on OPBs, especially 3-D structure of OBP/ligand complex, reveals more information on explaining what OBPs contribute to insect-specific and -selective olfaction.

$\mathrm{X}$-ray diffraction analysis and nuclear magnetic resonance (NMR) on crystal and solution of protein, respectively, are powerful tools for protein 3-D structure alone or with its ligand. To date, crystal or NMR structures of OBPs or PBPs, from more than 10 species belonging to four orders of insect, are available in Entrez's 3-D structure database at NCBI, and over 20 papers explaining their details are in Table 3.

All published structures show that both OBPs and PBPs consist of at least $6 \alpha$-helixes, which are held together by three disulfide bridges, cys1-cys3, cys2-cys5, and cys4-cys6, when only label cysteine residues form orderly disulfide bridges. In contrast, different mechanisms of ligand binding, release, and receptor recognition have been revealed by the diversity of key sites related to function, 3-D structure, and physicochemical property among OBPs.

$\mathrm{pH}$-dependent conformational change associated with change of binding affinity is reported to be common to OBPs in distinct insects. Moreover, it also shows a special character to different groups. For the moth, $\mathrm{pH}$ dependence has been demonstrated initially in Bombyx mori (Sandler et al., 2000). Asp-132 and Glu-141 are two well-conserved amino acid residues in moth PBPs, which form two salt bridges with protonated His-70 (Damberger et al., 2007; Xu and Leal, 2008) in both A. polyphemus and B. mori or His-80 (Xu et al., 2010) in Amyelois transitella and His-95, respectively, at low $\mathrm{pH}$ (4.5). The formation of two salt bridges together are referred to as histidine protonation switch (Xu et al., 2010), as they are demonstrated to pro- 
mote a C-terminal seventh $\alpha$-helix formation, and proposed to help these $\alpha$-helixes to disrupt the binding site of pheromone and replace the pheromone after a rapid ligand release to ORs. Deprotonation of the two His residues at $\mathrm{pH} 7$ has been demonstrated to cause the abolishment of salt bridges, promoting the withdrawal of the helix from the binding pocket and making the hydrophobic cavity available for sex pheromone.

Table 3. List of registered odorant-binding protein (OBP) 3-D structures (published with original paper)

\begin{tabular}{|c|c|c|c|c|}
\hline OBPs name & Species & Accession No. (MMDB ID) & Reference & Determined by \\
\hline CquiOBP1 & Culex quinquefasciatus & 85954 & Mao et al., 2010 & $\begin{array}{l}\text { X-ray } \\
1.3 \AA\end{array}$ \\
\hline AaegOBP1 & Aedes aegypti & 78632 & Leite et al., 2009 & $\begin{array}{l}\text { X-ray } \\
1.85 \AA \\
\end{array}$ \\
\hline OBP76a (LUSH) & Drosophila melanogaster & $\begin{array}{l}32732 \\
62291-62295\end{array}$ & Thode et al., 2008 & $\begin{array}{c}\text { X-ray } \\
2 \AA\end{array}$ \\
\hline OBP76a (LUSH) & Drosophila melanogaster & 46137 & Laughlin et al., 2008 & $\begin{array}{l}\text { X-ray } \\
1.4 \AA\end{array}$ \\
\hline$\overline{\text { OBP76a (LUSH) }}$ & Drosophila melanogaster & $23921-23924$ & Kruse et al., 2003 & $\begin{array}{l}\text { X-ray } \\
2.04 \AA\end{array}$ \\
\hline Atrapbp1 & Amyelois transitella & 79681 & Xu et al., 2010 & NMR \\
\hline BmorGobp2 & Bombyx mori & $75865-75871$ & Zhou et al., 2009 & $\begin{array}{l}\text { X-ray } \\
1.5 \AA\end{array}$ \\
\hline $\mathrm{BmPBP}$ & Bombyx mori & 21174 & Lee et al., 2002 & NMR \\
\hline$\overline{\mathrm{BmPBP}}$ & Bombyx mori & 18041 & Horst et al., 2001 & NMR \\
\hline$\overline{\mathrm{BmPBP}}$ & Bombyx mori & 15475 & Sandler et al., 2000 & $\begin{array}{l}\text { X-ray } \\
1.8 \AA\end{array}$ \\
\hline BmorPBP & Bombyx mori & $\begin{array}{l}45969 \\
45970\end{array}$ & Lautenschlager et al., 2007 & $\begin{array}{l}\text { X-ray } \\
2.01 \AA\end{array}$ \\
\hline ApolPBP & Antheraea polyphemus & 35432 & Zubkov et al., 2005 & NMR \\
\hline ApolPBP & Antheraea polyphemus & 26871 & Mohanty et al., 2004 & NMR \\
\hline ApolPBP1 & Antheraea polyphemus & 60086 & Damberger et al., 2007 & NMR \\
\hline ASP1 & Apis mellifera & $\begin{array}{l}78405-78407 \\
64848-64850 \\
61456 \\
64867 \\
64870\end{array}$ & Pesenti et al., 2008 & $\begin{array}{l}\text { X-ray } \\
1.75 \AA\end{array}$ \\
\hline$\overline{\mathrm{ASP} 1}$ & Apis mellifera & $\begin{array}{l}71369-71372 \\
72879-72884\end{array}$ & Pesenti et al., 2009 & $\begin{array}{c}\text { X-ray } \\
1.6 \AA\end{array}$ \\
\hline$\overline{\mathrm{ASP} 2}$ & Apis mellifera & 34972 & Lescop et al., 2001 & NMR \\
\hline$\overline{\text { LmaPBP }}$ & Rhyparobia maderae & $\begin{array}{l}24088 \\
24093 \\
24102\end{array}$ & Lartigue et al., 2003 & $\begin{array}{l}\text { X-ray } \\
1.7 \AA ̊\end{array}$ \\
\hline
\end{tabular}

$\mathrm{NMR}=$ nuclear magnetic resonance.

For the mosquito, AgamOBP1, AaegOBP1 and CquiOBP1 are highly conserved OBPs expressed in Anopheles gambiae, Aedes aegypti and Culex quinquefasciatus, respectively. Their affinities for ligands are also pH-dependent (Wogulis et al., 2006; Leite et al., 2009; Mao et al., 2010), whereas a C-terminal loop lacks a C-terminus region, which is also identical to Lma PBP with a direct end after the sixth helix in Leucophaea maderae (Lartigue et al., 2003) comparing with moth OBPs, folding into an extra $\alpha$-helix at low $\mathrm{pH}$. Hydrogen bonds involving the $\mathrm{C}$-terminal loop, which forms part of the binding pocket, will be broken at low $\mathrm{pH}$ and make the loop open, releasing further ligands. Under several conditions, dimers dynamically trap pheromone at high concentrations to mitigate the stimulus saturation (Hon- 
son et al., 2003). This has been previously suggested, and almost all determined OBP crystal structures show a dimeric structure. However, the fashion of CquiOBP1/(5R,6S)-6-acetoxy5-hexadecanolide (MOP) was found to be novel in C. quinquefasciatus (Mao et al., 2010). Helices 4 and 5 not only form a hydrophobic tunnel but also a dimeric interface, which is also identified in AgamOBP1 and AaegOBP1. The lactone head of the MOP binds in the central cavity of CquiOBP1 and its tail is buried in the tunnel created by the dimmer, which shows no hydrogen bonds, but exclusively hydrophobic and van der Waals interactions. In solution, the binding is retained even after the dimmer is dissociated.

For the honey bee, the affinity of another OBP, the antennal-specific protein 1 (ASP1), for 9-keto-2(E)-decenoic acid (9-ODA) is opposite to the pH-dependent affinity of the insects mentioned above (Pesenti et al., 2008, 2009). At low pH (4), ASP1 binds to 9-ODA with higher affinity than at neutral $\mathrm{pH}$. This apparent paradox is explainable, considering that under physiological environment $\mathrm{pH}$ is not categorically acidic, basic or neutral, but it is different from lo$\mathrm{cal}$ microenvironments in any organism. It seems that $\mathrm{pH}$ dependence of ligand release has been adapted to local $\mathrm{pH}$ around the membrane of the OBP's corresponding neuron cell. The Asp35 residue is demonstrated to be essential for the formation of dimmer, which has 10 -fold lower affinity for ligand at neutral pH than monomeric ASP1 at pH 4 (Pesenti et al., 2009).

Ligand-dependent conformational change is another mechanism of ligand release (Mohl et al., 2002; Zhou et al., 2004b; Lautenschlager et al., 2005; Laughlin et al., 2008). It has been demonstrated that it plays a role in at least two different ways. BmorPBP keeps unavailable the conformation with an ordered C-terminal helix inside the binding pocket when the ligand is absent, even at neutral $\mathrm{pH}$ (high $\mathrm{pH}$ ) (Lautenschlager et al., 2005). For LUSH, it shows a binding affinity with more than one ligand (Kim et al., 1998; Xu et al., 2005), but it mediates the activation of or67d through a conformational change only when cVA is present. The interaction between Phe121 of LUSH and cVA triggers LUSH-activated conformation transformation by disrupting a salt bridge between Asp118 and Lys87, and then the activated LUSH is able to independently stimulate T1 neuron as a ligand with or without the presence of cVA (Laughlin et al., 2008).

\section{PHYSIOLOGICAL CHARACTERISTICS}

To stimulate olfactory neuron, hydrophobic odorants such as pheromones need carriers to help them to pass through the aqueous hemolymph in which the neuron is bathed. The first OBP as a putative pheromone-binding protein, ApolPBP1, was identified in the polyphemus moth, A. polyphemus (Vogt and Riddiford, 1981). All the OBPs under olfactory reception investigation are specifically expressed or at least highly expressed in insect olfactory organs and more functions of OBPs have been proposed to date. They are triggers of an activated corresponding receptor with the ligand as the $\mathrm{OBP} /$ ligand complex; deactivation or protection from enzymatic degradation of the pheromone, and a solubilizer and transporter of specific ligands. There are some experimental or model proofs for the functions based on OBP-binding properties as well as 3-D structure studies.

\section{SOLUBILIZER AND TRANSPORTER OF SPECIFIC LIGAND}

Some OBPs are required as solubilizer and/or transporter and/or specific-ligand selector like filter in insect olfactory perception. 
Although pheromone-binding capability of OBPs has been demonstrated in various species since 1981 (Vogt and Riddiford, 1981), the first indication that OBPs solubilize ligands and are required for olfactory perception in vivo originated from Van den Berg and Ziegelberger (1991). These authors showed that replacing the lymph in relatively empty sensilla of A. polyphemus, the induced-pheromone electrophysiological response was abolished.

Several investigations have indicated that the final specific perception to some ligands is contributed by recognition and selection of the OBPs located around ORs, but not ORs. In the moth, A. polyphemus, ApolPBP1 and ApolPBP2 bound to two sex pheromones (E,Z)-6,11hexadecadienyl acetate $(\mathrm{ACl})$ and (E,Z)-6,11-haxadecadienal (AL), respectively. However, the exchanged combination of ACl/ApolPBP2 and AL/ApolPBP1 excited neuron cells corresponded with PBP, but no longer with their specific ligands (Pophof, 2002). The same team later obtained a similar result in B. mori (Pophof, 2004).

Another remarkable finding is that the transgenic Drosophila, lacking the lush gene, was reported to be anosmic to the male pheromone cVA (Xu et al., 2005). Furthermore, or67d from Drosophila, missexpressed in other trichoid neurons, conferred cVA sensitivity in the presence of LUSH (Ha and Smith, 2006).

All the above indicate that some OBPs select ligand for downstream ORs as a ligand selector or filter, as well as, transporters in insect olfactory perception, which contributes to insect-specific olfactory perception.

However, some investigations have shown that OBPs are not necessary to mediate the interaction between ligands and ORs. Odorants could directly stimulate several receptors expressed in selected cell lines (Wetzel et al., 2001; Hallem et al., 2004). Compared with insect fast behavioral reaction (milliseconds), the systems mentioned above showed much slower response speed (several seconds), which was reviewed by Pelosi et al. (2006). These indicate that some ligands do not need OBPs as carriers but as solubilizers in lymph, leaving the obligation of olfactory specificity to the corresponding downstream ORs.

With further research, some OBPs reviewed in this section were also found to play a direct role in chemical signal transmission.

\section{ACTIVATION OF CORRESPONDING RECEPTOR THROUGH OBP/LIGAND COMPLEX}

To date, available information remains limited but it reveals an interesting diversity of probable mechanisms of how the OBP/ligand complex interacts with downstream OR.

\section{pH-DEPENDENT OBP/LIGAND COMPLEX RELEASE TO ORS}

OBPs may be involved as ligand selectors, transporters or deactivators after triggering olfactory signal transduction but they are not required for a trigger event, which means OBP/ligand complex will release its ligand after they arrive at specific ORs. This process may occur by a $\mathrm{pH}$ change, as released pheromone independently activates ORs. $\mathrm{pH}$-dependence was investigated first to explain "extra" peaks shown by NMR from a highly pure BmorPBP expressed in B. mori. Its tertiary structure exhibited a conformational transition between $\mathrm{pH}$ 5 and 6 (Wojtasek and Leal, 1999). Then, it became clear that a single form exists at $\mathrm{pH}$ below 4.9, whereas it will change into another form at $\mathrm{pH}$ above 6 (Damberger et al., 2000). 
BmorPBP binds to bombykol when it is in the lymph, whose $\mathrm{pH}$ is 6.5 (Kaissling and Thorson, 1980), but not when at the surface of dendrites, which has a lower $\mathrm{pH}$ induced by a negatively charged surface (Blomquist and Vogt, 2003). The occurrence of pH-dependent conformational change was strongly supported by structural biology (see the last Section).

\section{ACTIVATED OBPs INDEPENDENTLY STIMULATE NEURON}

As mentioned above, LUSH is required for cVA-induced behavior and normal cVA sensitivity of the T1 neurons (Xu et al., 2005; Ha and Smith, 2006). Further research has shown more details in this process. LUSH activated by cVA plays a role as ligand to activate its neuron (Laughlin et al., 2008) (see more in the last section). Sensory neuron membrane proteins (SNMPs) and or67d are required in this process. Here, PBP changes its conformation after binding to the ligand, and PBP/ligand complex mediates the related neuron activation in vivo.

\section{OBP/LIGAND COMPLEX RELEASE TO SNMPS}

The results of other studies have indicated another interaction pattern among LUSH/ cVA complex, SNMPs and or67d (Benton et al., 2007; Jin et al., 2008). They concluded that SNMP may act downstream from OBP (LUSH) and upstream from odorant receptors (or67d) in the generation of action potentials. And another olfactory receptor, HR13, shares less than $15 \%$ amino acid identity with or67d, and could be activated by a similar pheromone of cVA, which has suggested that it is the fatty acid-derived hydrocarbon tail common to these pheromones that needs SNMP, which is a conserved protein among species. In other words, as a conserved protein, SNMP but not ORs may interact with ligand released by OBPs here.

\section{PHEROMONE DEACTIVATION AND PROTECTION FROM ENZYMATIC DEGRADATION}

The expected pheromone half-life (in the range of $1 \mathrm{~s}$ ) is about 300 -fold shorter than the half-life of the pheromone measured on the living antenna (4-5 min). Here, an unclear process (degradation or inactivation) must exist in the organism to stops pheromone from stimulating receptor neuron. It was hypothesized that fast inactivation of pheromones is achieved by pheromone-degrading enzymes (Kaissling, 1972). However, the enzymatic degradation in vivo has been considered too slow (on a minute timescale) (Kasang et al., 1988) to account for the fall of the receptor potential (Kaissling, 2001). It has also been suggested that the

discrepancy between data from in vivo and in vitro experiments is due to the involvement of PBPs avoiding pheromone degradation (Kaissling, 2001). This result has been shown in vitro (Vogt and Riddiford, 1986; Kaissling, 2009). Major attention on modeling has been devoted to the unknown mechanism of odorant deactivation, and modeling also helps to understand the multiple functions of the PBP (Kaissling, 2001). A study suggested that the fall of the receptor potential after stimulus offset is not caused by pheromone degradation and that pheromone deactivation must occur and could be associated with PBP. This study is a combination of model N (Kaissling, 1998a, 2001), model R (Kaissling, 1998a,b) and it is compared with another model in which deactivation is a spontaneous, non-enzymatic process (Kaissling, 2009). More data (structural and kinetic) are available from B. mori and A. polyphemus. 
Increased PBP-binding capacity at high pheromone concentrations was observed, and it has been suggested to play a role of trapping pheromone for mitigating the stimulus saturation (Honson et al., 2003). Considering a suggestion in the elephant (Lazar et al., 2002), Vogt (2005) proposed a conjecture that some odor-OBP complexes may sequester ligand as a deactivator. Together with the high concentration of OBP, which is up to 10 $\mathrm{mM}$ in vivo (Klein, 1987), the protection of ligand from enzyme degradation and the ability of transformation from different conformations, it is worth considering that OBPs can be a candidate for ligand deactivator. The process of pheromone deactivation is an essential postulate that needs to be experimentally verified.

\section{PERSPECTIVES FOR APPLIED RESEARCH}

Making the insect olfactory molecular mechanisms clearer aids in a better understanding of behaviors of various agricultural pests and pathogenic insects. Moreover, it would be useful to find novel tools for pest control strategies. OBPs are the functional component located at the start of olfactory signal transmission, and insect OBPs belong to a distinct protein family from other organisms. Target insect OBPs as a point of penetration to stop or interfere with insect olfactory conception will alter their reproduction or feeding and, therefore, it will artificially avoid risky behaviors and help natural enemies detect their preys. On the other hand, it will prevent threats for humans or animals and is also ecofriendly. Insect OBPs generally show their sensitivity and selectivity to at least a group of species-specific ligands but they also show their plasticity in some cases. For example, $B$. mori PBP1 has higher affinity for non-pheromone than for its natural pheromone (Hooper et al., 2009). A translational research combining molecular basis of olfaction and chemical ecology, using CquiOBP1 as a molecular target to identify its unnatural ligands, has contributed to the commercially available oviposition attractant for C. quinquefasciatus (Leal et al., 2008), which is a mixture of nonanal and trimethylamine. It can be concluded that OBP is the target of transgenic plant (Beale et al., 2006) synthesizing the aphid alarm pheromone, E $\beta F$, as E $\beta F$-binding OBP (Apis OBP3) has been identified in A. pisum.

\section{WHAT MORE}

Multiple OBPs encoded by overlapping genes obp99a, obp99b, and obp99d contribute to the same odorant reception but show different olfactory response independent of acetophenone (promotion or inhibition) in Drosophila (Wang et al., 2010). Here, different OBPs interact with the same ligand but induce a different response, which indicates a more complicated unclear interaction pattern among OBPs or between OBP/ligand complex and ORs.

Additionally, $o b p$ genes contribute to different responses to the same odorant. Obp57e is proposed to be responsible for interspecies differences of response to hexanoic acid contained in the ripe fruit of $M$. citrifolia, which is the toxin to D. melanogaster and D. simulans, but harmless to $D$. sechelliaheld. However, the fact that obp57e ORF shows no alteration in different species suggests that the interspecies difference of host plant preference is in gene expression rather than in the structure of gene product (Matsuo et al., 2007). Further, polymorphisms including single-nucleotide polymorphisms (SNPs) of obp genes contribute to individual variation olfactory behavior (Keller and Vosshall, 2007; Wang et al., 2007, 2010). 


\section{CONCLUSION}

Following the conventional approach, homology cloning is an available strategy to identify OBP in different species that share the same semiochemical reception. It is worth paying more attention to modeling in order to enrich and develop comparisons of the very complicated physiology condition of insect olfactory system in vivo.

Although in the past 20 years many studies have contributed to the understanding of insect OBPs, further knowledge of insect olfactory perception is still lacking. The major puzzle is how exactly OBPs play their roles in insect olfactory perception. On the contrary, available information, such as diverging primary sequences coupled with selective expression; numbers of intraspecies OBPs; diverse but specific combinational patterns of OBP/ligand complexes; different interactions between OBP/ligands and transmembrane proteins (ORs and SNMPs), and the dimer of OBPs, indicate that insect OBPs may have adapted from different levels to contribute to insect-sensitive and -specific odorant perception.

\section{ACKNOWLEDGMENTS}

Research supported by the projects "The National Natural Science Foundation of China" (\#30971920); "National S and T Major Project" (\#2009ZX08012-007B); The International Cooperation Project between China and Belgium (\#2010DFA32810), and Inter-University Targeted Project between Belgium and China (PIC SHANDONG) and "The Earmarked Fund for Modern Agro-Industry Technology Research System" (\#nycytx-03).

\section{REFERENCES}

Beale MH, Birkett MA, Bruce TJ, Chamberlain K, et al. (2006). Aphid alarm pheromone produced by transgenic plants affects aphid and parasitoid behavior. Proc. Natl. Acad. Sci. U. S. A. 103: 10509-10513.

Benton R, Sachse S, Michnick SW and Vosshall LB (2006). Atypical membrane topology and heteromeric function of Drosophila odorant receptors in vivo. PLoS Biol. 4: e20.

Benton R, Vannice KS and Vosshall LB (2007). An essential role for a CD36-related receptor in pheromone detection in Drosophila. Nature 450: 289-293.

Bette S, Breer H and Krieger J (2002). Probing a pheromone binding protein of the silkmoth Antheraea polyphemus by endogenous tryptophan fluorescence. Insect Biochem. Mol. Biol. 32: 241-246.

Blomquist GJ and Vogt RG (2003). Insect Pheromone Biochemistry and Molecular Biology. In: The Biosynthesis and Detection of Pheromones and Plant Volatiles (Blomquist GJ and Vogt RG, eds.). Elsevier Academic Press, London, 3-18.

Buck L and Axel R (1991). A novel multigene family may encode odorant receptors: a molecular basis for odor recognition. Cell 65: 175-187.

Campanacci V, Krieger J, Bette S, Sturgis JN, et al. (2001). Revisiting the specificity of Mamestra brassicae and Antheraea polyphemus pheromone-binding proteins with a fluorescence binding assay. J. Biol. Chem. 276: 20078-20084.

Clyne PJ, Warr CG, Freeman MR, Lessing D, et al. (1999). A novel family of divergent seven-transmembrane proteins: candidate odorant receptors in Drosophila. Neuron 22: 327-338.

Damberger F, Nikonova L, Horst R, Peng G, et al. (2000). NMR characterization of a pH-dependent equilibrium between two folded solution conformations of the pheromone-binding protein from Bombyx mori. Protein Sci. 9: 1038-1041.

Damberger FF, Ishida Y, Leal WS and Wuthrich K (2007). Structural basis of ligand binding and release in insect pheromone-binding proteins: NMR structure of Antheraea polyphemus PBP1 at pH 4.5. J. Mol. Biol. 373: 811-819.

Foret $\mathrm{S}$ and Maleszka R (2006). Function and evolution of a gene family encoding odorant binding-like proteins in a social insect, the honey bee (Apis mellifera). Genome Res. 16: 1404-1413.

Francis F, Vandermoten S, Verheggen F, Lognay G, et al. (2005a). Is the (E)-farnesene only volatile terpenoid in aphids? J. Appl. Entomol. 129: 6-11.

Francis F, Martin T, Lognay G and Haubruge E (2005b). Role of (E)-beta-farnesene in systematic aphid prey location by 
Episyrphus balteatus larvae (Diptera: Syrphidae). Eur. J. Entomol. 102: 431-436.

Friedrich RW and Korsching SI (1997). Combinatorial and chemotopic odorant coding in the zebrafish olfactory bulb visualized by optical imaging. Neuron 18: 737-752.

Gao Q, Yuan B and Chess A (2000). Convergent projections of Drosophila olfactory neurons to specific glomeruli in the antennal lobe. Nat. Neurosci. 3: 780-785.

Gong DP, Zhang HJ, Zhao P, Xia QY, et al. (2009). The odorant binding protein gene family from the genome of silkworm, Bombyx mori. BMC Genomics 10: 332.

Ha TS and Smith DP (2006). A pheromone receptor mediates 11-cis-vaccenyl acetate-induced responses in Drosophila. J. Neurosci. 26: 8727-8733.

Hallem EA, Nicole FA, Zwiebel LJ and Carlson JR (2004). Olfaction: mosquito receptor for human-sweat odorant. Nature 427: 212-213.

Hekmat-Scafe DS, Scafe CR, McKinney AJ and Tanouye MA (2002). Genome-wide analysis of the odorant-binding protein gene family in Drosophila melanogaster. Genome Res. 12: 1357-1369.

Hildebrand JG and Shepherd GM (1997). Mechanisms of olfactory discrimination: converging evidence for common principles across phyla. Annu. Rev. Neurosci. 20: 595-631.

Honson N, Johnson MA, Oliver JE, Prestwich GD, et al. (2003). Structure-activity studies with pheromone-binding proteins of the gypsy moth, Lymantria dispar. Chem. Senses 28: 479-489.

Hooper AM, Dufour S, He X, Muck A, et al. (2009). High-throughput ESI-MS analysis of binding between the Bombyx mori pheromone-binding protein BmorPBP1, its pheromone components and some analogues. Chem. Commun. 5725-5727.

Horst R, Damberger F, Luginbühl P, Güntert P, et al. (2001). NMR structure reveals intramolecular regulation mechanism for pheromone binding and release. Proc. Natl. Acad. Sci. U. S. A. 98: 14374-14379.

Jacobs SP, Liggins AP, Zhou JJ, Pickett JA, et al. (2005). OS-D-like genes and their expression in aphids (Hemiptera: Aphididae). Insect Mol. Biol. 14: 423-432.

Jin X, Ha TS and Smith DP (2008). SNMP is a signaling component required for pheromone sensitivity in Drosophila. Proc. Natl. Acad. Sci. U. S. A.105: 10996-11001.

Kaissling KE (1972). Kinetic Studies of Transduction in Olfactory Receptors of Bombyx Mori. In: Int. Symp. Olfaction and Taste IV (Schneider D, ed.). Wissenschaftl Verlagsges, Stuttgart, 207-213.

Kaissling KE (1998a). Flux detectors versus concentration detectors: two types of chemoreceptors. Chem. Senses 23: 99-111.

Kaissling KE (1998b). Pheromone deactivation catalyzed by receptor molecules: a quantitative kinetic model. Chem. Senses 23: 385-395.

Kaissling KE (2001). Olfactory perireceptor and receptor events in moths: a kinetic model. Chem. Senses 26: 125-150.

Kaissling KE (2009). Olfactory perireceptor and receptor events in moths: a kinetic model revised. J. Comp Physiol. A Neuroethol. Sens. Neural Behav. Physiol. 195: 895-922.

Kaissling KE and Thorson J (1980). Insect Olfactory Sensilla: Structure, Chemical and Electrical Aspects of the Functional Organization. In: Receptors for Transmitters, Hormones and Pheromones in Insects (Sattelle DB, Hall LM and Hildebrand JG, eds.). Elsevier, Amsterdam, 261-282.

Kasang G, von Proff L and Nicholls M (1988). Enzymatic conversion and degradation of sex pheromones in antennae of the male silkworm moth Antheraea polyphemus. Z. Naturforsch. C Biosci. 43c: 275-284.

Keller A and Vosshall LB (2007). Influence of odorant receptor repertoire on odor perception in humans and fruit flies. Proc. Natl. Acad. Sci. U. S. A. 104: 5614-5619.

Kim MS, Repp A and Smith DP (1998). LUSH odorant-binding protein mediates chemosensory responses to alcohols in Drosophila melanogaster. Genetics 150: 711-721.

Klein U (1987). Sensillum-lymph proteins from antennal olfactory hairs of the moth Antheraea polyphemus (Saturniidae). Insect Biochem. 17: 1193-1204.

Kruse SW, Zhao R, Smith DP and Jones DN (2003). Structure of a specific alcohol-binding site defined by the odorant binding protein LUSH from Drosophila melanogaster. Nat. Struct. Biol. 10: 694-700.

Kurtovic A, Widmer A and Dickson BJ (2007). A single class of olfactory neurons mediates behavioural responses to a Drosophila sex pheromone. Nature 446: 542-546.

Laissue PP and Vosshall LB (2008). The olfactory sensory map in Drosophila. Adv. Exp. Med. Biol. 628: 102-114.

Lartigue A, Gruez A, Spinelli S, Riviere S, et al. (2003). The crystal structure of a cockroach pheromone-binding protein suggests a new ligand binding and release mechanism. J. Biol. Chem. 278: 30213-30218.

Laughlin JD, Ha TS, Jones DN and Smith DP (2008). Activation of pheromone-sensitive neurons is mediated by conformational activation of pheromone-binding protein. Cell 133: 1255-1265.

Lautenschlager C, Leal WS and Clardy J (2005). Coil-to-helix transition and ligand release of Bombyx mori pheromone- 
binding protein. Biochem. Biophys. Res. Commun. 335: 1044-1050.

Lautenschlager C, Leal WS and Clardy J (2007). Bombyx mori pheromone-binding protein binding nonpheromone ligands: implications for pheromone recognition. Structure 15: 1148-1154.

Lazar J, Greenwood DR, Rasmussen LE and Prestwich GD (2002). Molecular and functional characterization of an odorant binding protein of the Asian elephant, Elephas maximus: implications for the role of lipocalins in mammalian olfaction. Biochemistry 41: 11786-11794.

Leal WS, Chen AM, Ishida Y, Chiang VP, et al. (2005). Kinetics and molecular properties of pheromone binding and release. Proc. Natl. Acad. Sci. U. S. A. 102: 5386-5391.

Leal WS, Barbosa RM, Xu W, Ishida Y, et al. (2008). Reverse and conventional chemical ecology approaches for the development of oviposition attractants for Culex mosquitoes. PLoS One 3: e3045.

Lee D, Damberger FF, Peng G, Horst R, et al. (2002). NMR structure of the unliganded Bombyx mori pheromone-binding protein at physiological pH. FEBS Lett. 531: 314-318.

Leite NR, Krogh R, Xu W, Ishida Y, et al. (2009). Structure of an odorant-binding protein from the mosquito Aedes aegypti suggests a binding pocket covered by a pH-sensitive "Lid". PLoS One 4: e8006.

Lescop E, Briand L, Pernollet JC, Van Heijenoort C, et al. (2001). Letter to the Editor: 1H, 13C and 15N chemical shift assignment of the honeybee odorant-binding protein ASP2. J. Biomol. NMR 21: 181-182.

Mao Y, Xu X, Xu W, Ishida Y, et al. (2010). Crystal and solution structures of an odorant-binding protein from the southern house mosquito complexed with an oviposition pheromone. Proc. Natl. Acad. Sci. U. S. A. 107: 19102-19107.

Matsuo T, Sugaya S, Yasukawa J, Aigaki T, et al. (2007). Odorant-binding proteins OBP57d and OBP57e affect taste perception and host-plant preference in Drosophila sechellia. PLoS Biol. 5: e118.

Mohanty S, Zubkov S and Gronenborn AM (2004). The solution NMR structure of Antheraea polyphemus PBP provides new insight into pheromone recognition by pheromone-binding proteins. J. Mol. Biol. 337: 443-451.

Mohl C, Breer H and Krieger J (2002). Species-specific pheromonal compounds induce distinct conformational changes of pheromone binding protein subtypes from Antheraea polyphemus. Invert. Neurosci. 4: 165-174.

Mori K, Nagao H and Yoshihara Y (1999). The olfactory bulb: coding and processing of odor molecule information. Science 286: 711-715.

Neuhaus EM, Gisselmann G, Zhang W, Dooley R, et al. (2005). Odorant receptor heterodimerization in the olfactory system of Drosophila melanogaster. Nat. Neurosci. 8: 15-17.

Novotny V, Basset Y, Miller SE, Weiblen GD, et al. (2002). Low host specificity of herbivorous insects in a tropical forest. Nature 416: 841-844.

Pelletier J and Leal WS (2009). Genome analysis and expression patterns of odorant-binding proteins from the Southern House mosquito Culex pipiens quinquefasciatus. PLoS One 4: e6237.

Pelosi P, Pisanelli AM, Baldaccini NE and Gagliardo A (1981). Binding of [3H]-2-isobutyl-3-methoxypyrazine to cow olfactory mucosa. Chem. Senses 6: 77-85.

Pelosi P, Baldaccini NE and Pisanelli AM (1982). Identification of a specific olfactory receptor for 2-isobutyl-3methoxypyrazine. Biochem. J. 201: 245-248.

Pelosi P, Zhou JJ, Ban L and Calvello M (2006). Soluble proteins in insect chemical communication. Cell. Mol. Life Sci. 63: 1658-1676.

Pesenti ME, Spinelli S, Bezirard V, Briand L, et al. (2008). Structural basis of the honey bee PBP pheromone and $\mathrm{pH}-$ induced conformational change. J. Mol. Biol. 380: 158-169.

Pesenti ME, Spinelli S, Bezirard V, Briand L, et al. (2009). Queen bee pheromone binding protein $\mathrm{pH}$-induced domain swapping favors pheromone release. J. Mol. Biol. 390: 981-990.

Pophof B (2002). Moth pheromone binding proteins contribute to the excitation of olfactory receptor cells. Naturwissenschaften 89: 515-518.

Pophof B (2004). Pheromone-binding proteins contribute to the activation of olfactory receptor neurons in the silkmoths Antheraea polyphemus and Bombyx mori. Chem. Senses 29: 117-125.

Qiao H, Tuccori E, He X, Gazzano A, et al. (2009). Discrimination of alarm pheromone (E)-beta-farnesene by aphid odorant-binding proteins. Insect Biochem. Mol. Biol. 39: 414-419.

Sandler BH, Nikonova L, Leal WS and Clardy J (2000). Sexual attraction in the silkworm moth: structure of the pheromone-binding-protein-bombykol complex. Chem. Biol. 7: 143-151.

Sato K, Pellegrino M, Nakagawa T, Nakagawa T, et al. (2008). Insect olfactory receptors are heteromeric ligand-gated ion channels. Nature 452: 1002-1006.

Silbering AF and Benton R (2010). Ionotropic and metabotropic mechanisms in chemoreception: "chance or design"? EMBO Rep. 11: 173-179.

Strausfeld NJ and Hildebrand JG (1999). Olfactory systems: common design, uncommon origins? Curr. Opin. Neurobiol. 9: 634-639. 
Tegoni M, Campanacci V and Cambillau C (2004). Structural aspects of sexual attraction and chemical communication in insects. Trends Biochem. Sci. 29: 257-264.

Thode AB, Kruse SW, Nix JC and Jones DN (2008). The role of multiple hydrogen-bonding groups in specific alcohol binding sites in proteins: insights from structural studies of LUSH. J. Mol. Biol. 376: 1360-1376.

Uchida N, Takahashi YK, Tanifuji M and Mori K (2000). Odor maps in the mammalian olfactory bulb: domain organization and odorant structural features. Nat. Neurosci. 3: 1035-1043.

Van den Berg MJ and Ziegelberger G (1991). On the function of the pheromone binding protein in the olfactory hairs of Antheraea polyphemus. J. Insect Physiol. 37: 79-85.

Vogt RG (2005). Molecular Basis of Pheromone Detection in Insects. In: Comprehensive Insect Physiology, Biochemistry, Pharmacology and Molecular Biology (Gilbert L, Latro G and Gill S, eds.). Elsevier, London, 753-804.

Vogt RG and Riddiford LM (1981). Pheromone binding and inactivation by moth antennae. Nature 293: 161-163.

Vogt RG and Riddiford LM (1986). Pheromone Reception: A Kinetic Equilibrium. In: Mechanisms in Insect Olfaction (Payne T, Birch M and Kennedy C, eds.). Clarendon Press, Oxford, 201-208.

Vosshall LB, Amrein H, Morozov PS, Rzhetsky A, et al. (1999). A spatial map of olfactory receptor expression in the Drosophila antenna. Cell 96: 725-736.

Vosshall LB, Wong AM and Axel R (2000). An olfactory sensory map in the fly brain. Cell 102: 147-159.

Wang P, Lyman RF, Shabalina SA, Mackay TFC, et al. (2007). Association of polymorphisms in odorant-binding protein genes with variation in olfactory response to benzaldehyde in Drosophila. Genetics 177: 1655-1665.

Wang P, Lyman RF, Mackay TF and Anholt RR (2010). Natural variation in odorant recognition among odorant-binding proteins in Drosophila melanogaster. Genetics 184: 759-767.

Wetzel CH, Behrendt HJ, Gisselmann G, Stortkuhl KF, et al. (2001). Functional expression and characterization of a Drosophila odorant receptor in a heterologous cell system. Proc. Natl. Acad. Sci. U. S. A. 98: 9377-9380.

Wicher D, Schafer R, Bauernfeind R, Stensmyr MC, et al. (2008). Drosophila odorant receptors are both ligand-gated and cyclic-nucleotide-activated cation channels. Nature 452: 1007-1011.

Wogulis M, Morgan T, Ishida Y, Leal WS, et al. (2006). The crystal structure of an odorant binding protein from Anopheles gambiae: evidence for a common ligand release mechanism. Biochem. Biophys. Res. Commun. 339: 157-164.

Wojtasek H and Leal WS (1999). Conformational change in the pheromone-binding protein from Bombyx mori induced by $\mathrm{pH}$ and by interaction with membranes. J. Biol. Chem. 274: 30950-30956.

Xu P, Atkinson R, Jones DN and Smith DP (2005). Drosophila OBP LUSH is required for activity of pheromone-sensitive neurons. Neuron 45: 193-200.

Xu PX, Zwiebel LJ and Smith DP (2003). Identification of a distinct family of genes encoding atypical odorant-binding proteins in the malaria vector mosquito, Anopheles gambiae. Insect Mol. Biol. 12: 549-560.

$\mathrm{Xu}$ W and Leal WS (2008). Molecular switches for pheromone release from a moth pheromone-binding protein. Biochem. Biophys. Res. Commun. 372: 559-564.

Xu X, Xu W, Rayo J, Ishida Y, et al. (2010). NMR structure of navel orangeworm moth pheromone-binding protein (AtraPBP1): implications for $\mathrm{pH}-$ sensitive pheromone detection. Biochemistry 49: 1469-1476.

Zhou JJ, Huang W, Zhang GA, Pickett JA, et al. (2004a). "Plus-C" odorant-binding protein genes in two Drosophila species and the malaria mosquito Anopheles gambiae. Gene 327: 117-129.

Zhou JJ, Zhang GA, Huang W, Birkett MA, et al. (2004b). Revisiting the odorant-binding protein LUSH of Drosophila melanogaster: evidence for odour recognition and discrimination. FEBS Lett. 558: 23-26.

Zhou JJ, He XL, Pickett JA and Field LM (2008). Identification of odorant-binding proteins of the yellow fever mosquito Aedes aegypti: genome annotation and comparative analyses. Insect Mol. Biol. 17: 147-163.

Zhou JJ, Robertson G, He X, Dufour S, et al. (2009). Characterisation of Bombyx mori Odorant-binding proteins reveals that a general odorant-binding protein discriminates between sex pheromone components. J. Mol. Biol. 389: 529-545.

Zhou JJ, Field LM and He XL (2010a). Insect odorant-binding proteins: do they offer an alternative pest control strategy? Outlooks Pest Manag. 21: 31-34.

Zhou JJ, Vieira FG, He XL, Smadja C, et al. (2010b). Genome annotation and comparative analyses of the odorant-binding proteins and chemosensory proteins in the pea aphid Acyrthosiphon pisum. Insect Mol. Biol. 19 (Suppl 2): 113-122.

Zubkov S, Gronenborn AM, Byeon IJ and Mohanty S (2005). Structural consequences of the pH-induced conformational switch in A. polyphemus pheromone-binding protein: mechanisms of ligand release. J. Mol. Biol. 354: 1081-1090. 